\title{
O026. An abnormal transduction of the chromatic stimuli from the outer to the inner retinal layers may contribute to cause photophobia in migraine
}

\author{
Gianluca Coppola ${ }^{1 *}$, Luisella Corso ${ }^{2}$, Antonio Di Renzo ${ }^{1}$, Antonello Fadda ${ }^{3}$, Francesco Martelli' \\ Cherubino Di Lorenzo ${ }^{4}$, Vincenzo Parisi ${ }^{1}$, Jean Schoenen ${ }^{5}$, Benedetto Falsini ${ }^{6}$, Francesco Pierelli ${ }^{2}$
}

From Abstracts from the 1st Joint ANIRCEF-SISC Congress

Rome, Italy. 29-31 October 2015

\section{Background}

Recent experimental evidence points out a possible involvement of outer and inner retinal layers in hypersensitivity of migraine patients to light stimuli. To investigate the short-wavelength-sensitive (S) and the medium/longwavelength-sensitive $(\mathrm{ML})$ cone photoreceptors of the visual pathways in migraine without aura (MO) patients between attacks and in healthy volunteers (HV) by using yellow-blue (Y-B) or red-blue (R-B) visual flicker stimuli.

\section{Methods}

Square-wave focal electroretinograms (FERGs) were recorded in $22 \mathrm{MO}$ patients and $20 \mathrm{HV}$. For each randomly presented flicker stimulation protocol (Y-B or R-B), 600 sweeps $(4 \mathrm{~Hz}$ repetition rate) were recorded and partitioned in 6 blocks of 100. Fourier analysis allowed extracting from the FERG data the fundamental $(1 \mathrm{~F})$ and the second harmonic (2F) components (amplitude and phase) that are related respectively to outer and inner retinal activity. Usual headache severity and photophobia during migraine were scored on a 0 to 10 visual analogue scale.

\section{Results}

When compared to $\mathrm{HV}, \mathrm{MO}$ patients had an advanced $1 \mathrm{~F}$ phase but normal amplitude in all blocks of Y-B FERG. In MO patients, the self-rated intensity of ictal photophobia was positively correlated with attack frequency $(r=0.571$, $\mathrm{p}=0.01)$, headache severity $(\mathrm{r}=0.508, \mathrm{p}=0.03), 1 \mathrm{~F} \mathrm{Y}-\mathrm{B}$ phase (all blocks $\mathrm{r}=0.487, \mathrm{p}=0.04$ ), $1 \mathrm{~F} \mathrm{R}-\mathrm{B}$ phase $(\mathrm{r}=0.521, \mathrm{p}=0.03), 2 \mathrm{~F} \mathrm{Y}-\mathrm{B}$ amplitude (all $\mathrm{r}=0.610$, $\mathrm{p}<0.01)$, habituation slope $(\mathrm{r}=0.686, \mathrm{p}<0.01)$, and $2 \mathrm{~F}$ R-B phase $(r=0.526, p=0.03)$.

\section{Conclusions}

These results suggest that an abnormal signal transduction from the outer to the inner retinal layers could contribute to the mechanisms by which light causes pain or discomfort during the migraine headache.

Written informed consent to publication was obtained from the patient(s).

\section{Authors' details}

'G.B. Bietti Foundation IRCCS, Department of Neurophysiology of Vision and Neuro-ophthalmology, Rome, Italy. "Sapienza" University of Rome Polo Pontino, Department of medico-surgical sciences and biotechnologies, Latina, Italy. ${ }^{3}$ Istituto Superiore di Sanità, Dipartimento Tecnologie e Salute, Rome, Italy. ${ }^{4}$ Don Carlo Gnocchi Onlus Foundation, Milan, Italy. ${ }^{5}$ Headache Research Unit, Department of Neurology-CHR Citadelle, University of Liège, Belgium. ${ }^{6}$ Catholic University of S. Cuore, Rome, Italy.

Published: 28 September 2015

\section{doi:10.1186/1129-2377-16-S1-A54}

Cite this article as: Coppola et al: O026. An abnormal transduction of the chromatic stimuli from the outer to the inner retinal layers may contribute to cause photophobia in migraine. The Journal of Headache and Pain 2015 16(Suppl 1):A54.

\footnotetext{
*Correspondence: gianluca.coppola@gmail.com

'G.B. Bietti Foundation IRCCS, Department of Neurophysiology of Vision and Neuro-ophthalmology, Rome, Italy

Full list of author information is available at the end of the article
} 